\title{
Reallabore in Theorie und Praxis: Reflexion des Forschungsdesigns im Hinblick auf die nachhaltige Transformation urbaner Räume
}

\author{
Kerstin Meyer, Dajana Esch, Maria Rabadjieva \\ Eingegangen: 31. Juli 2020 - Angenommen: 9. Juni 2021 - Online veröffentlicht: 8. Juli 2021
}

\begin{abstract}
Zusammenfassung
Im Rahmen der nachhaltigen Transformation von Kommunen werden Reallabore seit 2015 vermehrt als Instrumente in Forschungsprojekten eingesetzt. Deren Umsetzungen wurden bis jetzt jedoch nur wenig in der Wissenschaft reflektiert. In diesem Beitrag werden Reallabore als kooperatives Instrument zur nachhaltigen Transformation urbaner Räume betrachtet. Anhand einer vergleichenden Analyse der Projekte „Bottrop 2018+“, „KuDeQua“ und „ProUrban“ wird der Frage nachgegangen, inwiefern Reallabore als theoretisches Konzept in der Praxis greifen. Trotz des gemeinsamen theoretischen Hintergrunds weisen die Projekte wesentliche Unterschiede in der Thematik, räumlichen Reichweite, Einbeziehung der Praxisakteure sowie der Prozesse und Vorgehensweisen auf. Basierend auf dem Vergleich werden Chancen, Herausforderungen und mögliche Probleme bei der Umsetzung von Reallaboren herausgearbeitet.
\end{abstract}

Schlüsselwörter: Reallabor - Transformation - Urbane Räume - Stadt- und Regionalentwicklung - Aktionsforschung

Kerstin Meyer, Institut Arbeit und Technik, Munscheidstraße 14, 45886 Gelsenkirchen, Deutschland kmeyer@iat.eu

Dajana Esch, Institut Arbeit und Technik, Munscheidstraße 14, 45886 Gelsenkirchen, Deutschland Esch@iat.eu

Maria Rabadjieva, Institut Arbeit und Technik, Munscheidstraße 14, 45886 Gelsenkirchen, Deutschland rabadjieva@iat.eu

(c) (1) () 2021 Meyer; licensee oekom verlag. This Open Access article is published under a Creative Commons Attribution 4.0 International License.
Real-world laboratories in theory and practice: Reflection of the research design regarding the sustainable transformation of urban spaces

\begin{abstract}
As part of sustainable transformation of municipalities, realworld laboratories have been increasingly used as instruments in research projects since 2015. However, the academic reflection of their implementation has so far been scarce. This paper views real laboratories as a cooperative instrument for the sustainable transformation of urban spaces. It investigates the research question, to what extend realworld laboratories work as a theoretical concept in practice. The paper undertakes a comparative analysis of the projects "Bottrop 2018+", "KuDeQua" and "ProUrban", where real-world laboratories present the central methodology. Despite their common theoretical background, the projects show significant differences in the subject matter, spatial scope, practitioners' involvement, processes and procedures. Through the comparison, opportunities, challenges and possible problems in implementing real-world laboratories are worked out.
\end{abstract}

Keywords: Real-world laboratory - Transformation - Urban areas - Urban planning - Aaction research

\section{Einleitung}

Spätestens seit der Konferenz der Vereinten Nationen im Jahr 1992 in Rio de Janeiro gilt nachhaltige Entwicklung als globales Leitbild, das im Jahr 2002 auf der Konferenz in Johannesburg weiter konkretisiert wurde (Hens/Nath 2003: 7). In der Folge wurden zahlreiche Strategien zur Implementierung des Leitbildes in die Stadt- und Regionalentwicklung erarbeitet. Zugleich war die Forschung aufgefordert, sich 
dem Thema verstärkt zu widmen. So fordert das Gutachten des Wissenschaftlichen Beirats Globale Umweltveränderungen (WBGU 2011: 341) eine auf gesellschaftliche Transformation in Richtung Nachhaltigkeit fokussierte Forschung. Das Gutachten postuliert das, was im Nachgang der Konferenzen kritisiert wurde: Indikatoren und Handlungsfelder sowie deren Wechselwirkungen sind stark isoliert voneinander betrachtet und die Diskussion um die Implementierung von Nachhaltigkeitsstrategien in die Stadt- und Regionalentwicklung ist konzeptionell unzureichend abgesichert worden (von Hauff/Kleine 2005: 1).

In der heutigen Diskussion lassen sich Herausforderungen aufgrund der komplexen Wirkungsgefüge nicht mehr isoliert betrachten (Georg/Quaas 2018: 2). So beinhalten die Nachhaltigkeitsforschung und die transformative Wissenschaft ein erweitertes Wissensverständnis: Neben dem Systemwissen (was ist?) bedarf es des Zielwissens (wohin soll sich etwas entwickeln?) und Transformationswissens (wie kann diese Veränderung erreicht werden?) (Georg/Quaas 2018: 2). Zur Generierung von Systemwissen ist der Einbezug kontextualisierter Formen von Alltagswissen der Akteure ebenso relevant wie das Verständnis darüber, dass Transformationswissen nicht allein analytisch oder modellbasiert erfolgen kann, sondern anwendungsorientiertes Experimentieren (Erfahrungswissen) bedarf (Georg/Quaas 2018: 2).

Reallabore gelten als besonders geeignet für die Generierung von Transformationswissen, da sie Grenzen klassischer Beobachtungs- und modellgestützter Methoden überwinden, indem sie Raum zum Ausprobieren und Aushandeln nachhaltiger Zukunftsthemen bieten (Alcántara/ Lindner/Löwe et al. 2018: 9). Der Aspekt der Nachhaltigkeit als Ziel transformativer Forschung wird im deutschsprachigen Raum implizit oder explizit vorausgesetzt (Defila/Di Giulio 2018: 11). So orientiert sich transformative Forschung an konkreten gesellschaftlichen Herausforderungen und ist durch einen expliziten Interventionsanspruch gekennzeichnet. ${ }^{1}$ Waren Reallabore im Sinne der transformativen Forschung zunächst auf den Aspekt der Nachhaltigkeit fokussiert, wird der Begriff nun auch breiter verwendet (BMWi 2019: 7).

Trotz des vermehrten Einsatzes in der Praxis befindet sich die Methodik der Reallabore noch in einer Entwicklungsphase. Die wissenschaftlichen und praktischen Erfahrungen aus den letzten Jahren gilt es zu diskutieren, um das Konzept weiterzuentwickeln. An dieser Stelle setzt der vorliegende Beitrag mit folgender Forschungsfrage an: Inwiefern greifen Reallabore als theoretisches Konzept in der

\footnotetext{
1 Vgl. https://wupperinst.org/forschung/transformativeforschung/ (19.05.2019).
}

Praxis? Nach der Darstellung des Forschungsstandes in Kapitel 2 werden - anhand einer Operationalisierung der Charakteristik der Reallabore nach Schäpke, Stelzer, Bergmann et al. (2017) - drei Forschungsprojekte des Instituts Arbeit und Technik (IAT) reflektiert (Kapitel 3). Ferner wird untersucht, inwieweit die Reallabore dieser Projekte den Charakteristika gerecht werden. Durch die Gegenüberstellung von theoretischem Konzept und tatsächlicher praktischer Umsetzung (Kapitel 4) wird die Fundierung von Reallaboren als Methode diskutiert und es werden Schlussfolgerungen für Wissenschaft und Praxis formuliert (Kapitel 5 und 6).

\section{Forschungsstand}

Der Begriff des Reallabors überträgt den naturwissenschaftlichen Labor-Begriff auf die Analyse gesellschaftlicher und politischer Prozesse und erweitert diesen im Rahmen aktueller transformationsorientierter Nachhaltigkeitsdisziplinen auf einen sozialen Kontext (Wanner/Stelzer 2019: 1). Entstanden ist die Reallaborforschung aus der von Groß, Hoffmann-Riem und Krohn (2005) angelegten Idee des „Realexperimentes“, mit dem Interventionen in einen gesellschaftlichen Kontext bezeichnet wurden. Realexperimente in Reallaboren sind nicht in erster Linie darauf ausgerichtet, durch Forschung neues Wissen zu generieren (Groß/Hoffmann-Riem/Krohn 2005: 11). Vielmehr entsteht das ,neue“ Wissen bzw. Transformationswissen durch die Kombination des Wissens einer Vielzahl von Akteuren, die in soziale, ökologische und technische Gestaltungsprozesse eingebettet sind (Groß/Hoffmann-Riem/Krohn 2005; Grillitsch/Trippl 2014). Das entstandene Format der Reallabore gewann im deutschsprachigen Raum, aber auch im internationalen Kontext (z. B. living labs, (urban) transition labs) in den vergangenen Jahren an Bedeutung (Schäpke/ Stelzer/Bergmann et al. 2017: 1). Dabei wurden die Konturen des Formats durch wissenschaftliche Debatten und den zunehmenden Rückgriff auf anwendungsorientierte Forschungsbereiche kontinuierlich geschärft.

Es existieren mehrere Definitions- und Nutzungsansätze von Reallaboren in den unterschiedlichen gesellschaftlichen Bereichen (vgl. Grunwald 2015; Schäpke/Stelzer/Bergmann et al. 2017), wodurch es kaum Einschränkungen bezüglich der räumlichen Handlungsebenen von Reallaboren gibt. Es kann sich sowohl um eine ganze Region, eine Stadt, ein einzelnes Quartier oder auch ein ausgewähltes Setting handeln. Ebenso denkbar ist, dass Reallabore keinen physischen Ort haben, sondern sich beispielsweise an Branchen oder Wertschöpfungsketten orientieren. Ebenso vielfältig sind die in den Reallaboren bearbeiteten Themen.

Wir verstehen in diesem Beitrag Reallabore als ein Format, das transdisziplinäre und transformative Nachhaltig- 
keitsforschung miteinander kombiniert, einen starken Fokus auf gesellschaftliche, langfristige Lernprozesse aufweist und durch realweltliche Interventionen (Realexperimente) gefasst wird (Schäpke/Stelzer/Bergmann et al. 2017: 1, 19; WBGU 2016: 542). Ziel von Reallaboren ist es - ausgehend von einem gemeinsamen Problemverständnis -, ein vertiefendes Wissen über Dynamiken von Transformationsprozessen sowie kontextabhängiges, sozial robustes Wissen zu erlangen und Konzepte zu erproben, um die soziale Innovationskraft und Systeminnovationen zu befördern (Howaldt/ Schwarz 2010; Rose/Wanner/Hilger 2019). Somit werden Reallabore als möglicher Lösungsansatz zum Testen transformativer Ideen und als Schnittstelle zwischen Wissenschaft und Gesellschaft gesehen, um lokale und regionale Zukunftsprojekte anzustoßen. Dies stellt hohe Ansprüche sowohl an Wissenschaft als auch an Praxisakteure und bringt neue Herausforderungen in Bezug auf die Zusammenarbeit mit sich.

Vor dem Hintergrund divergierender Begriffsverständnisse sowie variierender Ansichten zum Aufbau, zur Methode und zu den Zielen (Beecroft/Trenks/Rhodius et al. 2018: 75) kann die Fortentwicklung des Reallabor-Ansatzes von einem Vergleich unterschiedlicher Reallabore profitieren. Solche Analysen aus Theorie und Praxis bilden immer noch die Ausnahme (vgl. Gonser/Eckart/Eller et al. 2019; Rose/ Wanner/Hilger 2019; Parodi/Waitz/Bachinger et al. 2020). Dieser Beitrag liefert eine detaillierte Analyse und regt darüber hinaus zur Diskussion an. Die in dem vielfältigen Diskurs über die Konturen des Reallabor-Ansatzes von Schäpke, Stelzer, Bergmann et al. (2017: 9-27) herausgearbeiteten Charakteristika - Transformationsanspruch, Transdisziplinarität, Realexperimente, Langfristigkeit und Übertragbarkeit sowie Evaluation - bieten einen Rahmen, anhand dessen die Reallabore verglichen werden können. Folgend wird die theoretische Fundierung der Charakteristika näher erläutert.

\subsection{Transformation}

Transformation steht für einen umfassenden Wandel politischer, ökonomischer, ökologischer, kultureller und gesellschaftlicher Teilsysteme und beinhaltet eine grundlegende Änderung gesellschaftlicher Systeme und Teilsysteme. Transitionen hingegen beschreiben den Übergang von einem Teilsystem in ein anderes (Welschhoff/Terstriep/ Seipel et al. 2017: 30). Eine Transformation resultiert aus mehreren Transitionen, die durch Nischeninnovationen (kleinräumig) oder Megatrends (gesellschaftliche oder natürliche Phänomene) ausgelöst werden können (Grießhammer/Brohmann 2015: 6-7). Transformation im Kontext von Reallaboren bedeutet, auf Veränderungen von Alltagskulturen und täglichen Routinen in Nischen hinzuarbeiten
(Parodi/Waitz/Bachinger et al. 2020: 284). Dabei zielen diese auf die Erzeugung von handlungsleitendem Wissen (WBGU 2011: 341), welches als Grundlage für die konkrete Gestaltung von Wandel dient. Dieses Wissen wird von Schäpke, Stelzer, Bergmann et al. (2017: 9) in Systemwissen (was ist?), Orientierungswissen (was (nicht) sein soll?) und Transformationswissen (wie gelangt man vom Ist-Zustand in den Soll-Zustand?) untergliedert. Reallabore zielen insofern gleichermaßen auf das Verstehen von Problemen und deren Bewertung sowie Lösungsmöglichkeiten und deren konkrete Ausgestaltung ab (Schäpke/Stelzer/Bergmann et al. 2017: 9). Mit diesem Anspruch reihen sie sich in die Methodik der transformativen Forschung ein, deren primärer Fokus das aktive und gezielte Anstoßen einer gesellschaftlichen Veränderung ist (Defila/Di Giulio 2018: $1 \mathrm{f}$.). Die Geschwindigkeit, mit der diese Transformation abläuft, kann dabei unterschiedlich sein (Grießhammer/Brohmann 2015: 6).

\subsection{Transdisziplinarität}

Transdisziplinarität ist in der Transformationsforschung ein zentraler Baustein. Beim akteursorientierten Ansatz werden im Rahmen der transdisziplinären Forschung neben den Wissenschaftlerinnen und Wissenschaftlern aus verschiedenen Disziplinen weitere, aus der Praxis stammende Akteure substanziell am Forschungsprozess beteiligt (Defila/De Giulio 2018: 10-11). Damit knüpft dieser Ansatz an die Aktions- und Interventionsforschung an (Parodi/Beecroft/Albiez et al. 2016: 9-10). Die Beteiligung kann im Projektteam oder als punktuelle Beteiligung im Reallaborverlauf erfolgen (Defila/De Giulio 2018: 10-11). Dies begründet eine Zusammenarbeit der Akteure auf mindestens zwei Ebenen: zum einen auf der Ebene des Projekts und zum anderen innerhalb der Reallabore (Beecroft/Trnks/Rhodius et al. 2018: 90). Die adressierten realweltlichen Probleme werden dabei in einem wechselseitigen, iterativen Lernprozess zwischen Wissenschaft und Gesellschaft in Ko-Design und Ko-Produktion bearbeitet (Lang/Wiek/Bergmann et al. 2012: 27; Schäpke/Stelzer/Bergmann et al. 2017: 19).

\subsection{Experimente}

Nach De Flander, Hahne, Kegler et al. (2014: 285) entfaltet sich die Wissensproduktion in Reallaboren in einem Spannungsfeld zwischen der klassischen Methode der Grundlagenforschung und der forschungsbasierten Unterstützung realweltlicher Transformationsprozesse im lokalen Raum. Aufgrund der starken Abhängigkeit von lokalem, kontextspezifischem Wissen, aber auch der hohen Komplexität der Nachhaltigkeitsherausforderungen bzw. der adressierten Probleme und des normativen Charakters bedarf es, 

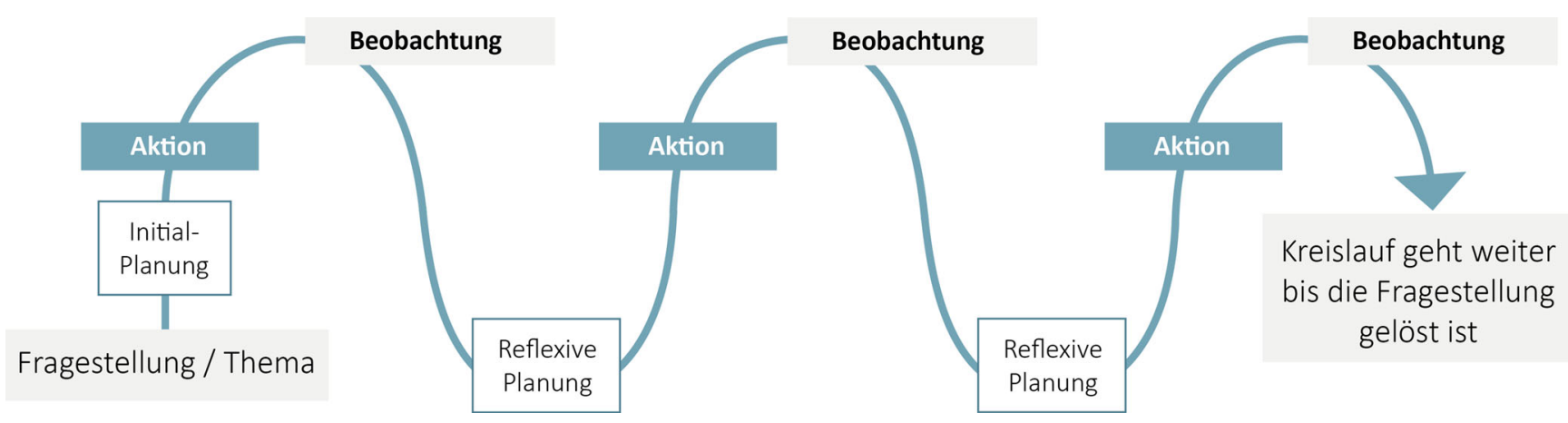

Abbildung 1 Der iterative Kreislauf der „Participatory Action Research“ Quelle: Eigene Darstellung nach Walter (2009: 153)

neben den traditionellen experimentellen Praktiken, neuer, offener Ansätze (Schäpke/Stelzer/Bergmann et al. 2017: 15). Die Arten des Experimentierens lassen sich nach $\mathrm{Ca}-$ niglia, Schäpke, Lang et al. (2017) anhand des Ausmaßes der Kontrolle und der Form des Wissens unterteilen: Im Hinblick auf die Art der Kontrolle sieht der in Reallaboren praktizierte Weg eine regelmäßige partizipative Kontrolle über das Experiment sowie seinen Kontext vor und erlaubt eine gemeinsame Gestaltung des Experiments und der Betrachtung des Kontextes durch die Akteure. Bezogen auf die Form des Wissens gilt insbesondere die Erzeugung von handlungsleitendem Problemlösungswissen als relevant (Schäpke/Stelzer/Bergmann et al. 2017: 15-16).

\subsection{Langfristigkeit und Übertragbarkeit}

Da Transformationsprozesse in der Regel über die Dauer der Forschungsprojekte hinausgehen, sollten Reallabore darauf abzielen, langfristige Experimentier- und Lösungswege aufzubauen. Die gewonnenen Erkenntnisse gilt es, in die Praxis und die Wissenschaft zurückzuspielen sowie auf andere Kontexte zu übertragen (Schäpke/Stelzer/Caniglia et al. 2018: 87). Diese Ziele werden von Schäpke, Stelzer, Bergmann et al. (2017) unter dem Charakteristikum „Langfristigkeit und Übertragbarkeit“ zusammengefasst. Während die Langfristigkeit primär auf die Verstetigung der gewonnenen Erkenntnisse im eigenen Kontext abstellt, zielt die Übertragbarkeit auf den Transfer dieser Erkenntnisse über den ursprünglichen Kontext hinaus.

Bei der Verstetigung von Reallaboren können nach Parodi (2020) zwei Aspekte betrachtet werden. Erstens können einzelne Reallaboreinheiten bzw. Realexperimente durch Empowerment und stabile Kooperationen langfristig etabliert werden. Dabei ist wichtig, transparent zu kommunizieren, was zu erwarten und was leistbar ist. Selbstausbeutung auf Teamseite gilt es genauso zu vermeiden wie die Überforschung der Zivilgesellschaft. Zudem sollten sich alle aktiv Beteiligten vorab mit einem potenziellen Scheitern auseinandersetzen, um keine ,verbrannte Erde` zu hinterlassen. Scheitern kann dabei als ein wesentlicher Bestandteil des Lernprozesses verstanden werden, da es die Suche nach neuen Problemlösungen stimuliert (Barth/Michelsen 2013: 114). Zweitens kann das Reallabor als Institution oder Labor transformativer Wissenschaft angelegt werden. Hierbei wird eine langfristige Kooperation und Infrastruktur zwischen Wissenschaft und einer praxisorientierten Organisation angestrebt, die die Realexperimente vor Ort umsetzt (z. B. Forschungsvorhaben TransZ $Z^{2}$ ). Insbesondere auf der Strukturebene können Reallabore längerfristige Impulse für den Aufbau und den Erhalt von Infrastrukturen und Prozesse geben (Schneidewind/Augenstein/Stelzer et al. 2018: 14).

Bei der Übertragbarkeit von Erkenntnissen stellt sich zunächst die Frage nach dem ,Wohin': Handelt es sich um einen anderen Ort, einen anderen Kontext (Schäpke/ Stelzer/Caniglia et al. 2018: 87) oder ein Upscaling (Schäpke/Stelzer/Bergmann et al. 2017: 25)? Generell gilt, dass die Wirkung von Reallaboren steigt, wenn diese aktuelle und ortsspezifische Themen behandeln. Dem gegenüber steht, dass durch die kontextabhängige und situationsbedingte Thematik der Grad der Übertragbarkeit sinkt (Parodi 2020). Neben der Nutzung der Ergebnisse im praktischen Kontext fließen diese in den wissenschaftlichen Diskurs zurück und ermöglichen so eine Weiterentwicklung von Theorien und Methoden (Schäpke/Stelzer/Bergmann et al. 2017: 25).

\subsection{Kontinuierliche Reflexion}

In Anlehnung an die „Participatory Action Research“ (Walter 2009) erfolgt in Reallaboren während und nach einer Aktion bzw. eines Experiments die Beobachtung und an-

\footnotetext{
2 https://www.transz.de (20.05.2021).
} 
schließend die reflexive Planung bzw. Reflexion. Die kontinuierliche methodologische Reflexion (vgl. Abbildung 1) bildet damit ein weiteres Kerncharakteristikum des Reallabors. Im Rahmen der „Participatory Action Research“ wird der Prozess solange wiederholt, bis eine zufriedenstellende Lösung für die Fragestellung gefunden wird.

Diese fünf Charakteristika - Transformation, Transdisziplinarität, Experimente, Langfristigkeit und Übertragbarkeit, Kontinuierliche Reflexion - werden nachfolgend genutzt, um drei Fallstudien von Projekten, in denen Reallabore umgesetzt wurden, zu vergleichen. Bevor die Ergebnisse des Vergleichs vertieft dargestellt und diskutiert werden, legt das nächste Kapitel die Methodik des Vergleichs dar.

\section{Methodik}

Der Gegenstand dieses Beitrags liegt in der Anwendung der vergleichenden Analyse von Fallstudien. Durch die systematische Gegenüberstellung von empirischen Tatbeständen ist es möglich zu lernen und das Wissen über die Anwendung und den Nutzen von Reallaboren zu erhöhen. Somit bestehen die Ziele des Vergleichens darin, die Unterschiede und Gemeinsamkeiten sowie funktionale Äquivalente der durchgeführten Reallabore zu erkennen, bestehende Klassifikationen und Typologien zu prüfen und weiterzuentwickeln sowie Prognosen für ein idealtypisches Reallabor aufzustellen (Lauth/Pickel/Pickel 2009: 28; Jahn 2011: 44).

Die vergleichende Analyse beruht auf der Konstruktion von drei „diszipliniert-konfigurativen“ (Verba 1967: 115) Fallstudien, die es erlauben, bestehende Annahmen zu testen (konfirmatorisch) und anhand der Analyse neue Annah- men aufzustellen (explorativ). Der Fokus des Vergleichs liegt dabei in der Überprüfung der kausalen und sozialen Mechanismen der Reallabore.

Ein Vergleich war nicht Teil der Projekte selbst, ist jedoch deshalb interessant, da die drei Projekte in Förderprogrammen des Bundesministeriums für Bildung und Forschung (BMBF) mit ähnlichen Zielen (,Nachhaltige Transformation urbaner Räume“ und „Kommunen innovativ“), gleichem Zeitraum und durch dieselbe Institution (Institut Arbeit und Technik - IAT), aber dort unabhängig voneinander von unterschiedlichen leitenden Wissenschaftlerinnen und Wissenschaftlern durchgeführt wurden. Wegen des gleichen Zeitraums der Projektkonzeption wurde die gleiche Literatur zur Reallaborforschung zugrunde gelegt. Jedoch variieren die Umsetzungszeiträume in den Projekten. Im Projekt „ProUrban“ waren die Reallabore der Kern des Vorhabens, das gesamte Projekt war auf die Durchführung der Reallabore ausgelegt. Im Projekt „Bottrop 2018+“ wurden Reallabore als Beteiligungsinstrument eingesetzt. Ähnlich verhielt es sich in „KuDeQua“: Ausgehend von der Erhebung der Problemlagen wurden daraus Konzepte in den Quartieren entwickelt und anschließend Umsetzungsmöglichkeiten erprobt. Dieser Unterschied spielt eine Rolle bezüglich der zeitlichen Umsetzung, die sich in den genannten Projekten zwischen anderthalb und drei Jahren bewegte. Das methodische Vorgehen war jedoch in allen drei Projekten ähnlich: In den Projektvorhabenbeschreibungen wurden erste Ziele und Konzepte für die Reallabore vordefiniert, die im Projektverlauf an lokale Bedürfnisse angepasst wurden. Dies ermöglicht eine Analyse der Unterschiede zwischen dem Projektvorhaben und der tatsächlichen Durchführung

Tabelle 1 Fallbeispiele

Projektname

Bottrop 2018+- Auf dem Weg zu
einer nachhaltigen und resilienten Wirtschaftsstruktur (Bottrop 2018+)

$\begin{array}{ll}\begin{array}{l}\text { Zeitraum } \\ \text { Förder- } \\ \text { programm }\end{array} & \begin{array}{l}\text { 2016-2019 + 2020-2021 } \\ \text { BMBF „Nachhaltige Transformation } \\ \text { Reallabor- } \\ \text { fokus }\end{array} \\ \begin{array}{l}\text { Gesamtstadt Bottrop - einzelne } \\ \text { Wertschöpfungsketten und Branchen } \\ \text { als Reallabore }\end{array} \\ \text { Ziel } & \begin{array}{l}\text { Gestaltung einer nachhaltigen und } \\ \text { resilienten Wirtschaftsstruktur durch } \\ \text { Partizipation }\end{array}\end{array}$

Zielgruppe
KuDeQua

QUARTIERSLABORE

KuDeQua - Kultur- und demographiesensible Entwicklung bürgerschaftlich getragener Finanzierungsund Organisationsmodelle für gesellschaftliche Dienstleistungen im Quartier (KuDeQua)

2017-2020

BMBF „Kommune innovativ“

Zwei Stadtteile in Dortmund als Reallabore

Entwicklung alternativer Finanzierungs- und Organisationsmodelle zur Entwicklung tragfähiger Geschäftsmodelle

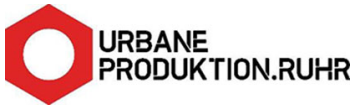

ProUrban - Produktion zurück in die Stadt?! (PU)

2016-2019 + 2020-2021

BMBF „Nachhaltige Transformation urbaner Räume“

Zwei Stadtteile in Bochum als Reallabore

Aufwertung von Stadtteilen durch urbane Produktion

Bürger/-innen, Kommune 
sowie den Vergleich der Erkenntnisse zwischen den drei Projekten.

Für den systematischen Vergleich wurde im ersten Schritt ein Fallstudienbericht-Template erstellt. Das Template trug dazu bei, die Rolle und das Verständnis von Reallaboren in der Projektstruktur (theoretischer Ansatz zur Methodik und Erwartung der einzelnen Akteursgruppen zu Beginn der Projekte) sowie die Erfahrungen aus der Umsetzung (durchgeführte Experimente und Maßnahmen, beteiligte Akteure sowie Prozessdynamiken) zu erfassen. Die Fallstudienberichte wurden anhand von projektspezifischen Texten und Dokumenten (Ergebnisberichte, Protokolle, Fragebögen und Interviews) von der jeweiligen Autorin ausgefüllt. Im zweiten Schritt wurden die fünf Charakteristika von den Autorinnen genutzt, um die Templates mithilfe von MaxQDA zu kodieren und auszuwerten. Tabelle 1 stellt die drei Fallbeispiele vor.

Der nachfolgende Vergleich der Reallabore erhebt auch vor dem Hintergrund, dass einzelne Fallstudien nicht für Verallgemeinerungen und Falsifikationen von Theorien geeignet sind (Lijphart 1971: 691), keinen Anspruch auf Allgemeingültigkeit. Weder sollen Besonderheiten überbewertet noch Ad-hoc-Erklärungen produziert werden. Dennoch erscheint die gewählte Methode zielführend, um die zuvor dargestellten Charakteristika von Reallaboren im Hinblick auf die tatsächliche Umsetzung zu untersuchen und gegebenenfalls zu erweitern, daraus Schlussfolgerungen über die Methodik der Reallabore zu ziehen und die Rolle der Wissenschaft zu beleuchten.

\section{Ergebnisse}

Die Ergebnisse der Analyse werden im Folgenden anhand der in Kapitel 2 erläuterten Charakteristika dargestellt und erörtert. Das Hauptergebnis jedes Punktes wird zu Beginn hervorgehoben.

\subsection{Transformation}

Reallabore können die „Große Transformation“ nicht erreichen, tragen aber in Nischen zur Transition von Teilsystemen bei.

Die drei analysierten Projekte sollten laut Ausschreibung der BMBF-Förderprogramme in den drei Städten einen Beitrag zur Transformation leisten, indem sie Transitionen innerhalb von Reallaboren, das heißt durch multiple NischenInnovationen, anstoßen. Der Vergleich der Projekte wurde von der Frage geleitet, inwieweit die transformative Zielsetzung erreicht und Transformationswissen generiert wurde. Tabelle 2 fasst die jeweilige Zielsetzung in den Projekten zusammen.

Die Generierung von Transformationswissen ist in unterschiedlichem Ausmaß gelungen. In Bottrop 2018+ wurden die Reallabore als ein dauerhaftes Beteiligungsinstrument der Wirtschaftsförderungstätigkeit über die Projektlaufzeit hinaus beibehalten und somit die Transition der Wirtschaftsförderungsstruktur angestoßen. In KuDeQua konnten konkrete langfristige Maßnahmen in der Projektlaufzeit nicht umgesetzt werden. Die Herausforderungen wurden von den Bürgerinnen und Bürgern zwar erkannt, die Zielgruppen - Kommunen und Bürger/-innen - durch Informations- und Aufklärungsmaßnahmen für notwendige Veränderungen sensibilisiert und mit diesen zum selbstständigen Handeln befähigt. In ProUrban ist es gelungen, die Verwaltung für das Thema „Urbane Produktion“ zu sensibilisieren und die Zivilgesellschaft zu aktivieren. Somit wurde die angestrebte Transition angestoßen. Die Fallstudien zeigen, dass eine räumliche Abgrenzung in Bezug auf den Anstoß von Transitionen durch Nischen-Innovationen keine Rolle spielt.

Die Wissenschaft hat in allen Projekten Systemwissen erzeugt, um die Themen im Fokus - nachhaltige Wirtschaftsförderung, nachhaltige Daseinsvorsorge und urbane Produktion - zu bearbeiten. Jedoch konnte das generierte Wissen in der Projektlaufzeit nicht in allen Fällen in konkrete Maßnahmen überführt werden (Transformationswissen). In ProUrban wurde beispielsweise eine langfristige Nutzung

Tabelle 2 Projektthemen und Inhalte zur Transition jeweiliger Teilsysteme

\begin{tabular}{|c|c|c|c|}
\hline & Bottrop 2018+ & KuDeQua & ProUrban \\
\hline Zielsetzung & $\begin{array}{l}\text { Veränderung von Routinen und Wirt- } \\
\text { schaftspraktiken. Transition des } \\
\text { Handelns von Wirtschaftsakteuren. } \\
\text { Strukturelle Veränderung durch Be- } \\
\text { teiligungsinstrumente (Reallabore) } \\
\text { als Teil der Wirtschaftsförderungs- } \\
\text { praktik. }\end{array}$ & $\begin{array}{l}\text { Transformation der Daseinsvorsorge } \\
\text { in der Kommune. Wiederbelebung } \\
\text { und Wiederaufbau von gesellschaftli- } \\
\text { chen Dienstleistungen mithilfe alter- } \\
\text { nativer Finanzierungs- und Organi- } \\
\text { sationsmodelle und Sensibilisierung } \\
\text { für neue Akteursstrukturen. }\end{array}$ & $\begin{array}{l}\text { Aufwertung von Stadtteilen durch } \\
\text { gemeinschaftliche urbane Produkti- } \\
\text { on. Umwidmung ungenutzter Immo- } \\
\text { bilien. Transition der Wertschätzung } \\
\text { gegenüber der Produktion in der } \\
\text { Stadt in den Teilsystemen Politik, } \\
\text { Verwaltung und Zivilgesellschaft. }\end{array}$ \\
\hline $\begin{array}{l}\text { Räumliche } \\
\text { Abgrenzung }\end{array}$ & $\begin{array}{l}\text { Stadt; kein physischer Raum, eher } \\
\text { thematisch: Wirtschaftsbranchen }\end{array}$ & $\begin{array}{l}\text { Stadtteil; physischer Raum (Ladenlo- } \\
\text { kal) als Ort }\end{array}$ & $\begin{array}{l}\text { Stadtteil; physischer Raum (Kirche, } \\
\text { Ladenlokal) als Ort }\end{array}$ \\
\hline
\end{tabular}


von Leerständen zunächst nur in einem Reallabor erfolgreich umgesetzt. Im zweiten Reallabor dagegen wurden die lokalen Akteure über die Bedeutung der Nutzung des Leerstandes sensibilisiert (Orientierungswissen), sodass in der Folgeförderung eine längerfristige, aktive Nutzung ausgelöst werden konnte. Ähnlich konnte in KuDeQua das Thema „Gründung“ aus Mangel an Gründungswilligen nicht weiterbearbeitet werden. Jedoch wurde das Wissen über die Gründe und Problemlagen möglicher Finanzierungsschwierigkeiten von schwach- bis unrentierlichen Unternehmungen im Bereich der Daseinsvorsorge vor Ort erweitert (Systemwissen). Ähnlich wiederum verlief es in Bottrop 2018+. Hieraus lässt sich schließen, dass mit Reallaboren ein gutes Instrument zur Verfügung steht, um System- und Orientierungswissen zu erzeugen. Dieses Wissen gilt es weiter zu operationalisieren.

Diese Ergebnisse zeigen deutlich, dass Reallabore keine große Transformation schaffen können, jedoch zu Transitionen in Teilsystemen beitragen.

\subsection{Transdisziplinarität}

Reallabore stellen Wissenschaft und Praxis vor neue Herausforderungen, die nur durch hohe Flexibilität und Wahrnehmung unterschiedlicher Rollen überwunden werden können.

Reallabore als Methode der transformativen Forschung werden konzipiert, durchgeführt und umgesetzt. Dies hebt die Fragen hervor, wer an diesen Prozessen beteiligt ist und was die Zusammenarbeit in den jeweiligen Phasen charakterisiert. Um die Transdisziplinarität vollständig zu evaluieren, wird in diesem Beitrag eine zweiteilige Betrachtung vorgenommen: einerseits wird die Zusammenarbeit zwischen Wissenschaft und Praxis innerhalb der Projektteams und andererseits zwischen dem Projektteam und der Zielgruppe evaluiert.

Tabelle 3 fasst die Dynamik der Zusammenarbeit in den drei Fallstudien zusammen und zeigt, dass in allen drei Städten die jeweilige Kommune feste Partnerin im Verbundprojekt ist. Somit kooperierten Wissenschaft und Praxis schon bei der Konzipierung des Gesamtvorhabens und der ein-

Tabelle 3 Beteiligte Akteure im Projektteam und in den Reallaboren

\begin{tabular}{|c|c|c|c|}
\hline & Bottrop 2018+ & KuDeQua & ProUrban \\
\hline Wissenschaft & $\begin{array}{l}\text { IAT, Faktor } 10 \text { - Institut für nachhal- } \\
\text { tiges Wirtschaften }\end{array}$ & IAT & IAT, Hochschule Bochum \\
\hline Kommune & $\begin{array}{l}\text { Stadt Bottrop, Amt für Wirtschafts- } \\
\text { förderung und Standortmanage- } \\
\text { ment }\end{array}$ & Stadt Dortmund, „nordwärts“ & $\begin{array}{l}\text { Stadt Bochum, Amt für Stadtpla- } \\
\text { nung und Wohnen, Wirtschaftsent- } \\
\text { wicklungs-Gesellschaft }\end{array}$ \\
\hline $\begin{array}{l}\text { Weitere Pra- } \\
\text { xisakteure im } \\
\text { Projektteam }\end{array}$ & - & NRW.Bank & Die Urbanisten e.V. \\
\hline $\begin{array}{l}\text { Weitere Pra- } \\
\text { xisakteure in } \\
\text { den Realla- } \\
\text { boren (inkl. } \\
\text { Zielgruppe) }\end{array}$ & $\begin{array}{l}\text { Unternehmen, Politik, IHK, Hand- } \\
\text { werkskammer, Verbände, Stadtver- } \\
\text { waltung, Hochschule Ruhr-West }\end{array}$ & $\begin{array}{l}\text { (organisierte) Zivilgesellschaft, Un- } \\
\text { ternehmen, Wirtschaftsförderung }\end{array}$ & $\begin{array}{l}\text { Stadtteilmanagement, IHK, Werbe- } \\
\text { gemeinschaft, Initiativen, Unter- } \\
\text { nehmen, soziokulturelle Zentren, } \\
\text { Politik, Zivilgesellschaft, später } \\
\text { LutherLAB e.V. }\end{array}$ \\
\hline Rollenverteilung & $\begin{array}{l}\text { Wissenschaft: } \\
\text { Begleitende, impulsgebende und } \\
\text { evaluierende Rolle } \\
\text { Kommune: } \\
\text { Gesamtkoordination, Vernetzung, } \\
\text { Kontaktpflege, Aufbau der Realla- } \\
\text { bore } \\
\text { Weitere Praxisakteure: } \\
\text { Umsetzung der Reallabore in } \\
\text { selbstdefinierten Rollen }\end{array}$ & $\begin{array}{l}\text { Wissenschaft: } \\
\text { Begleitende, beratende und impuls- } \\
\text { gebende Rolle } \\
\text { Kommune: } \\
\text { Gesamtkoordination, Vernetzung, } \\
\text { Kontaktpflege, Aufbau und Beglei- } \\
\text { tung der Reallabore } \\
\text { Weitere Praxisakteure: } \\
\text { Finanzierung und Organisation, } \\
\text { Analyse und Weiterentwicklung }\end{array}$ & $\begin{array}{l}\text { Wissenschaft: } \\
\text { Gesamtkoordination; analysieren- } \\
\text { de, konzipierende und evaluierende } \\
\text { Rolle } \\
\text { Kommune: } \\
\text { Vernetzung, Kontaktpflege, Öffent- } \\
\text { lichkeitsarbeit } \\
\text { Weitere Praxisakteure: } \\
\text { Aufbau und Begleitung der Realla- } \\
\text { bore }\end{array}$ \\
\hline Anmerkungen & $\begin{array}{l}\text { Die Selbstdefinierung der Rolle der } \\
\text { weiteren Praxisakteure außerhalb } \\
\text { des Projektteams hatte eine uner- } \\
\text { wünschte Dynamik von extensiver } \\
\text { Vor- und Nachbereitung seitens der } \\
\text { Kommune und Absprache mit den } \\
\text { Reallaborteilnehmenden zur Folge. }\end{array}$ & $\begin{array}{l}\text { Die Rollenzuschreibung innerhalb } \\
\text { des Projektes war zu Beginn defi- } \\
\text { niert, änderte sich im Verlauf des } \\
\text { Forschungsprojektes jedoch. In re- } \\
\text { gelmäßigen Teamsitzungen wurden } \\
\text { Rollenverteilungen und Aufgaben- } \\
\text { zuschreibungen - auch über die } \\
\text { zunächst vordefinierten hinweg - } \\
\text { neu aufgestellt. }\end{array}$ & $\begin{array}{l}\text { Die Praxisakteure im Team bekun- } \\
\text { deten ebenfalls Interesse, an der } \\
\text { Analyse und Konzeptentwicklung } \\
\text { beteiligt zu werden, was in einem } \\
\text { intensiven Austausch in regelmäßi- } \\
\text { gen Treffen und einer gemeinsam } \\
\text { genutzten Plattform mündete. }\end{array}$ \\
\hline
\end{tabular}


zelnen Reallabore. Die jeweilige Zielgruppe wurde in der Durchführungsphase eingebunden.

Zusammenarbeit im Projektteam: Den wissenschaftlichen Akteuren kommen in diesem Rahmen neben den traditionellen Aufgaben zusätzliche Funktionen zu, etwa als Botschafter, Promotoren oder ,Brückenbauer', in der Öffentlichkeitsarbeit oder bei der Aktivierung von Zielgruppen (Beecroft/Trenks/Rhodius et al. 2018: 90). Die Praxisakteure (unter anderem Kommunen), deren traditionelle Aufgabe die Identifikation lebensweltlicher Problemlagen ist sowie implizites und lokales (Praxis)wissen einzubringen ist, bekommen zusätzlich die Verantwortung als Multiplikatoren und gegebenenfalls die langfristige Trägerschaft der zu entwickelnden Strukturen und Lösungswege. Diese Rolle kann dahingehend erweitert werden, indem sie als Ko-Forschende einbezogen werden und die transdisziplinären Projekte im Ko-Design mitplanen, durchführen, Ergebnisse auswerten und integrieren (Beecroft/Trenks/Rhodius et al. 2018: 90). Die neudefinierten Rollen und Verantwortungen münden in einer herausfordernden Kooperation zwischen Wissenschaft und Praxis, die nur durch Kommunikation und basierend auf einer starken Vertrauensbasis gelingen kann (Kanders/Rösner 2006: 35).

Die Zusammenarbeit in transdisziplinären Teams entwickelt, abhängig vom Thema, von Persönlichkeiten und Arbeitsprozessen, eine eigene Dynamik und kann schwer verallgemeinert werden. Lütje-Klose und Urban (2014: 117) entwarfen mit ihrer Arbeit über transdisziplinäre Teams in der Lehre und Sozialarbeit eine vierstufige Skala, um die Zusammenarbeit zu klassifizieren:

- Ko-Aktivität: hohes Maß an Selbstbestimmung und additive Arbeitsteilung

- Koordination: höheres Maß an Absprachen, gelegentliche Teambesprechungen, grundsätzliche Zielsetzungen, getrennte Zuständigkeiten, eher additive Arbeitsteilung

- Kooperation: detaillierte Absprachen über Verantwortung und Aufgaben, regelmäßige Teambesprechungen, Möglichkeiten und Grenzen des/der Einzelnen wurden definiert, sodass persönliche Fähigkeiten eingebracht werden können

- Kollaboration: grundlegende Übereinstimmung der Werte und Ziele, hohes Maß an gegenseitigem Vertrauen, Rollen können sich überschneiden (Führungswechsel möglich), Gefühl, die Verantwortung gemeinsam zu tragen

Obwohl sich die Grenzen zwischen den Ausprägungen der vierstufigen Skala nicht trennscharf ziehen lassen, ermöglichen sie doch einen Vergleich der drei Fallstudien. Die Zusammenarbeit in Bottrop 2018+ weist einen koordinativen Charakter auf, während in KuDeQua eine Kooperation er- kennbar ist und ProUrban in Richtung Kollaboration geht. Dass die Zusammenarbeit in Bottrop 2018+ von der Koordination geprägt wurde, lag überwiegend an der stringenten Rollentrennung im Projektvorhaben, mehrfachem Personalwechsel und den ,eingeübten', nur schwer zu durchbrechenden Arbeitsweisen zwischen Stadtverwaltung und Unternehmerschaft. In KuDeQua hat sich aufgrund der Teamzusammensetzung ein kooperativer Modus entwickelt: Rollenverteilungen haben sich im Verlauf durch Absprache und Kommunikation verändert und verschoben. Solche kontextbezogenen Faktoren müssen frühzeitig adressiert werden, um den gewünschten Grad an Zusammenarbeit zu erreichen. Als ein möglicher Grund für das Zustandekommen der Kollaboration der Partner im Projekt ProUrban ist der Umstand zu nennen, dass sich das Team aus Berufsanfängerinnen und -anfängern mit ähnlichem Ausbildungshintergrund zusammensetzte, die über eine hohe kognitive Nähe (vgl. Boschma 2005) verfügten. Daneben könnten sich die durchgeführten Teambildungmaßnahmen (z. B. Kreativworkshop zur Entwicklung des Projektablaufs und einer gemeinsamen Vision) als förderlich erwiesen haben.

Eine klare Rollenverteilung der Projektpartner und die damit einhergehende Arbeitsteilung hat sich in allen drei Projekten als nicht förderlich für die Reallabore erwiesen. Kontinuierliche Absprachen, ein hohes Maß an gegenseitigem Vertrauen und offene Kommunikation zwischen den Beteiligten tragen wesentlich dazu bei, die Reallabore und damit intendierten Transitionen anzustoßen. Zu berücksichtigen ist, dass das Reallabor sowohl für Angestellte in der Kommunalverwaltung als auch für viele Wissenschaftlerinnen und Wissenschaftler eine neue Methode darstellt. Ein Lernprozess und hohe Flexibilität in der Arbeitsgestaltung, auch in Bezug auf die Rollenzuschreibung innerhalb des Teams, sind gefragt, auch wenn dies der Forschungslogik widerspricht. Hinzu kommt, dass Forschungsanträge zum Teil von anderen Personen geschrieben werden, die nicht zwingend in die Umsetzung involviert sind, sodass im Umsetzungsteam zunächst ein gemeinsames Verständnis erarbeitet werden muss.

Zusammenarbeit mit weiteren Akteuren in den Reallaboren: Wie oben ausgeführt, stellt das Reallabor als Instrument eine Interaktionsplattform zur Beteiligung und Ko-Kreation von Lösungen dar. Genauso wie bei der Zusammenarbeit im Team ist der reale Beteiligungsgrad nur schwer im Voraus einschätzbar. Die Projektvorhaben verlangen eine aktive Einbindung weiterer Akteure (,Zielgruppe“) in allen Phasen der Reallabormethodik. Wie ,aktive Beteiligung ' aber zu verstehen ist, bleibt offen. Die Literatur zu Ko-Kreationsprozessen (Arnstein 1969; Fung 2006; O'Hern/Rindfleisch 2010) differenziert in Abhängigkeit vom Machtgefüge und dem Ausmaß der Verantwortungsübernahme unterschiedli- 


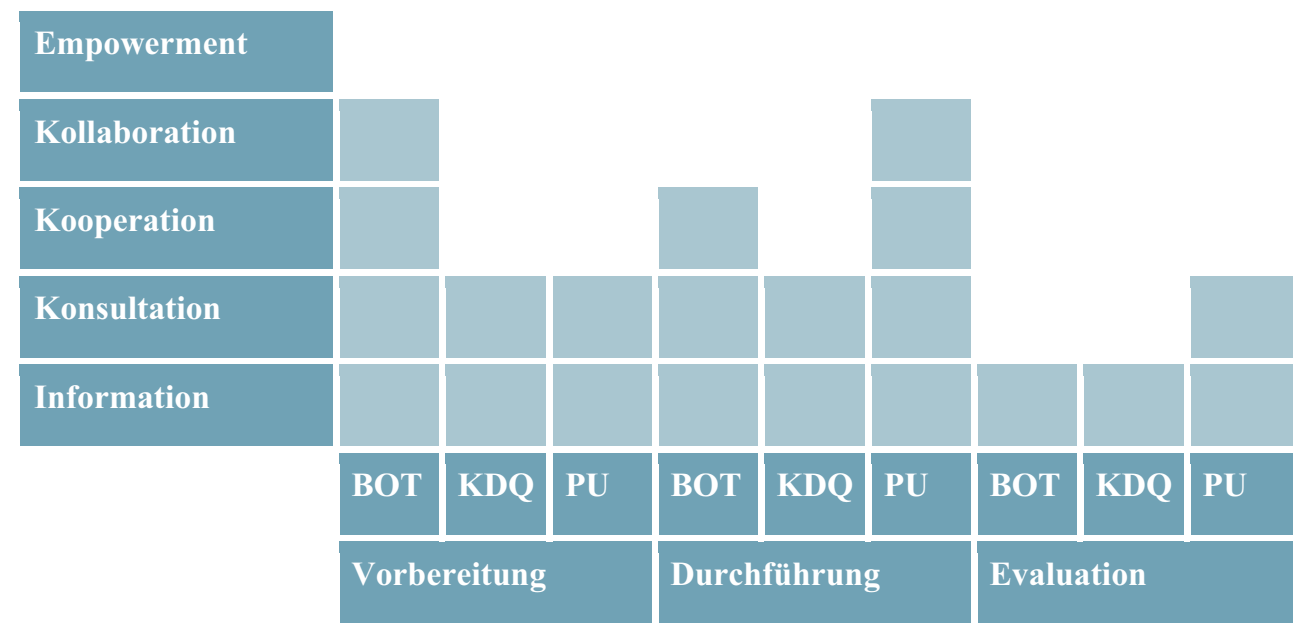

Abbildung 2 Beteiligungsgrad der Zielgruppe in den Projekten nach Phasen

Quelle: Eigene Darstellung, angelehnt an Stauffacher/Flüeler/Krütli et al. (2008: 414)

che Stufen der Beteiligung. In den Studien zu Reallaboren finden sich ähnliche Überlegungen (Stauffacher/Flüeler/ Krütli et al. 2008: 414), welche ebenfalls unterschiedliche Reallaborphasen berücksichtigen. Die Frage, inwieweit die Integration der Zielgruppe in den Reallaboren für alle Phasen der Umsetzung zumutbar ist, bedarf einer vertiefenden Diskussion. Die drei Fallstudien wurden zu diesem Zweck, angelehnt an die Klassifizierung von Stauffacher/Flüeler/ Krütli et al. (2008: 414), verglichen (vgl. Abbildung 2).

Die Erkenntnisse stellen die ,Ko'-Dimension von Reallaboren in Frage. Vielmehr lässt sich Beteiligung anhand eines Kontinuums von Information bis Empowerment charakterisieren. In allen drei Projekten gab es die Erwartung, dass die zu beteiligenden Gruppen aktiv im Prozess mitwirken werden. Bei Bottrop 2018+ war vorgesehen, dass die Unternehmens- und Wirtschaftsroutinen verändert und Konzepte wie Raum, Eigentum und Wirtschaftszweig überdacht werden. KuDeQua zielte mitunter darauf ab, dass die Bürgerinnen und Bürger bzw. gründungswillige Personen sich aktiv an dem Erhalt bzw. Wiederaufbau gesellschaftlich notwendiger Dienstleistungen im Quartier beteiligen. In ProUrban sollte ein freiwilliges Team, bestehend aus Bürgerinnen und Bürgern sowie weiteren interessierten Personen, die zwischengenutzten Räumlichkeiten zukünftig weiter betreiben. Diese Erwartungen wurden in allen Fällen nur bedingt erfüllt (vgl. Kapitel 4.3). Die bisherigen Erfahrungen zeigen, dass die einzelnen Akteure bzw. Akteursgruppen mit variierenden und zum Teil widersprüchlichen Zielsetzungen bzw. Erwartungen im Reallabor mitwirken. Es gilt, eine Balance zwischen divergierenden Zielen und Erwartungen zu finden. Die frühzeitige Integration der Zielgruppe in den Prozess der Konzeption (Vorbereitung) kann wesentlich dazu beitragen, potenzielle Konflikte und Problemlagen zu antizipieren. Jedoch wird dies durch die Lo- gik der öffentlichen Projektförderung erschwert. Projekte werden auf wissenschaftlicher Basis und vorheriger Erfahrung konzipiert, sodass die an der Umsetzung Beteiligten oft mit vordefinierten Bedarfen, Themen und Formaten in unbekannten Quartieren und Kommunen agieren müssen, die von den Praxisakteuren vor Ort nicht immer akzeptiert werden (z. B. in KuDeQua). Hinzu kommt die zeitliche Verzögerung zwischen Antragstellung und tatsächlichem Projektbeginn. Dies hat zur Folge, dass es notwendig wird, in den Projekten eine, zweite "Vorbereitungsphase, die als Teil des Reallabors zu verstehen ist (Abbildung 2 meint diese Vorbereitungsphase), mit starker Beteiligung der Zielgruppe durchzuführen. Dies ist in Bottrop und teilweise bei ProUrban geschehen. Damit einher geht die Anforderung, vordefinierte Ziele und Vorgehensweisen anzupassen, wofür die in der Regel maximal dreijährige Projektlaufzeit häufig nicht ausreicht. Gerade auch vor diesem Hintergrund haben sich aktiv Teilnehmende, welche als Botschafter agieren, als notwendig erwiesen, um die Experimente voranzutreiben (in Bottrop: Hochschule Ruhr-West oder einzelne Unternehmen; in ProUrban: Projektpartner/-innen; in KuDeQua: Bürger/-innen).

Der Vergleich der Zusammenarbeit auf den zwei Ebenen verdeutlicht, dass die Dynamik, das Einbeziehen und die Mitwirkung von weiteren Partnern in den unterschiedlichen Reallabor-Phasen stark variieren kann. Deshalb erscheinen bestimmte Kompetenzen, Fähigkeiten und Ressourcen, wie etwa offene Kommunikation, Moderation und Konfliktlösungen, Community-Bildung, geteilter Raum zum Austausch, offenes Denken wichtiger, als vordefinierte Rollenzuschreibungen. 
Tabelle 4 Umgesetzte Maßnahmen und Wissensgenerierung

\begin{tabular}{ll}
\hline $\begin{array}{l}\text { Maßnahmen } \\
\text { Maßnahmen }\end{array}$ & $\begin{array}{l}\text { Bottrop 2018+ } \\
\text { Verhaltensmuster ändern, neue Ge- } \\
\text { schäftsmodelle initiieren (nicht von } \\
\text { vornherein spezifiziert) }\end{array}$ \\
Umgesetzte & Umfrage zur Bedarfsermittlung (ein \\
Maßnahmen & $\begin{array}{l}\text { Reallabor), Workshop-Reihen (Schu- } \\
\text { lungen in allen drei Reallaboren), } \\
\text { Anschaffung von Technologie unter } \\
\text { der Leitung der Hochschule Ruhr- } \\
\text { West (ein Reallabor) }\end{array}$
\end{tabular}

Wissensgenerierung Kommune

Wissen über Verständnis und Wichtigkeit des Themas „Nachhaltigkeit“ in Unternehmen; methodisches Wissen

$\begin{array}{ll}\begin{array}{l}\text { Zielgruppe/ } \\ \text { Unternehmen }\end{array} & \begin{array}{l}\text { Sensibilisierung für das Thema } \\ \text { „Nachhaltigkeit“ }\end{array} \\ \text { Wissenschaft } & \begin{array}{l}\text { Methodisches und strukturelles } \\ \text { Wissen }\end{array}\end{array}$

Zielgruppe/ Sensibilisierung für das Thema Wissen

\section{KuDeQua}

Gründungsaktivität anstoßen bzw.

begleiten; Leerstand als Austauschraum zur Verfügung stellen; Sensibilisierung für alternative Daseinsvorsorgeleistungen

Veranstaltungsreihe (zu Best-Practice-Beispielen); Sensibilisierung in Bezug auf die Themen; Etablierung eines Quartierskoordinators

\begin{abstract}
ProUrban
Leerstände in Produktionsstätten umwandeln; Sensibilisierung für das Thema Urbane Produktion

Festivalisierung durch Zwischennutzung von leerstehenden Immobilien inklusive Workshop- und Veranstaltungsreihen zur Sensibilisierung. Der Erfolg des ersten Reallabors wirkte sich positiv auf das Anstoßen des zweiten Reallabors aus
\end{abstract}

Wissen über Notwendigkeit und Möglichkeiten des Erhalts und des Wiederaufbaus gesellschaftlich notwendiger Dienstleistungen im Quartier; Wissen über neue Kooperations- und Ko-Produktionsformen.

Sensibilisierung für das Thema „Daseinsvorsorge " und eigene Beteiligung

Methodisches und strukturelles Wissen
Verständnis und Potenziale des Themas „Urbane Produktion“ für die Aufwertung von Kommunen; methodisches Wissen über Ko-Produktion

Sensibilisierung und Verständnis für das Thema „Urbane Produktion“ und Bedeutung von eigenem Engagement Methodisches und strukturelles Wissen

\subsection{Experimente}

Der Unterschied zwischen Experiment und Maßnahmen bzw. Intervention bleibt oft unklar, was die Generierung von Transformationswissen erschwert.

Wie anfangs ausgeführt sind für die Experimente in Reallaboren das Ausmaß an Kontrolle und die Form des generierten Wissens von großer Relevanz. Im Vergleich der drei Fallstudien wurde der Frage gefolgt, in wie weit sich das $\mathrm{Ma} \beta$ an Kontrolle auf die Wissensgenerierung ausgewirkt hat. Tabelle 4 fasst die umgesetzten Maßnahmen und das generierte Wissen in den Fallstudien zusammen.

Es zeigt sich, dass die Reallabore partizipativ kontrolliert wurden. Die Offenheit gegenüber den zu beteiligenden Gruppen hat dazu geführt, dass zum Teil die erwünschten Ergebnisse nicht erreicht werden konnten. In allen drei Pro- jekten wurden Veranstaltungsreihen zur Information und Sensibilisierung für das anvisierte Thema als Hauptmaßnahmen umgesetzt. Während in ProUrban diese als Beteiligungsmethode genutzt wurden, können die Veranstaltungen und Workshops bei KuDeQua und Bottrop 2018+ als Ergebnis der Reallabore angesehen werden. Dies kann darauf zurückgeführt werden, dass in den einzelnen Teams Maßnahmen mit Realexperimenten gleichgesetzt wurden. Die Auswahl der Maßnahmen war in beiden Projekten sehr stark von den zu beteiligenden Gruppen abhängig. Da in KuDeQua Gründungswillige und Bürger/-innen fehlten, die aktiv in die Umsetzung gehen, war es nicht möglich, entsprechende Handlungen anzustoßen und zu begleiten. Ähnlich haben sich die Wirtschaftsakteure in Bottrop für greifbare Maßnahmen entschieden, in denen sie einen direkten Mehrwert für das eigene Handeln sehen. Die durchgeführ-

Tabelle 5 Reallabore über die Projekte hinaus (Verstetigung)

\begin{tabular}{|c|c|c|c|}
\hline & Bottrop 2018+ & KuDeQua & ProUrban \\
\hline $\begin{array}{l}\text { Zeitraum } \\
\text { der Real- } \\
\text { labore }\end{array}$ & $\begin{array}{l}\text { Alle gleichzeitig: konzipiert 2017; } \\
2018 \text { bis heute }\end{array}$ & Beide von 2018 bis 2020 & $\begin{array}{l}\text { Reallabor 1: 09/2017-heute; Realla- } \\
\text { bor 2: 05-12/2019 und 04/2020- } \\
\text { heute }\end{array}$ \\
\hline $\begin{array}{l}\text { Ergebnis - } \\
\text { Verstetigte } \\
\text { Struktur }\end{array}$ & $\begin{array}{l}\text { Anerkennung und Etablierung von } \\
\text { Reallaboren als Beteiligungsinstru- } \\
\text { ment und Umsetzungsebene in der } \\
\text { Wirtschaftsförderungstätigkeit vom } \\
\text { Amt für Wirtschaftsförderung und } \\
\text { von den Unternehmen }\end{array}$ & $\begin{array}{l}\text { Etablierung und Besetzung eines } \\
\text { Quartiersmanagements in einem der } \\
\text { Modellquartiere, Sensibilisierung für } \\
\text { das Thema sowohl bei Kommunen } \\
\text { als auch bei zivilgesellschaftlichen } \\
\text { Akteuren }\end{array}$ & $\begin{array}{l}\text { Seit } 2017 \text { Sonderpreis Urbane } \\
\text { Produktion in Gründungswettbe- } \\
\text { werb etabliert; Vereinsgründung } \\
\text { LutherLAB 2018; Vereinsgründung } \\
\text { WatWerk } 2020\end{array}$ \\
\hline
\end{tabular}


Tabelle 6 Verwertung der Ergebnisse aus den Reallaboren (Übertragbarkeit)

\begin{tabular}{|c|c|c|c|}
\hline & Bottrop 2018+ & KuDeQua & ProUrban \\
\hline Skalierung & $\begin{array}{l}\text { Eine Ausweitung hat im Projektzeit- } \\
\text { raum nicht stattgefunden, da die } \\
\text { Reallabore auf Branchen und The- } \\
\text { men fokussierten, deren Erkennt- } \\
\text { nisse sich nicht auf andere Bereiche } \\
\text { in Bottrop übertragen ließen. }\end{array}$ & $\begin{array}{l}\text { Eine Ausweitung der Re- } \\
\text { allabor-Aktionen hat nicht } \\
\text { stattgefunden. }\end{array}$ & $\begin{array}{l}\text { In Wattenscheid fand eine Skalierung statt, indem } \\
\text { der Ort des Reallabors in einen größeren verlagert } \\
\text { wurde. Bei der Skalierung der Gesamtstadt Bo- } \\
\text { chum lag der Fokus auf der Innenstadt. Deutsch- } \\
\text { landweite Diskussionen zum Thema urbane Pro- } \\
\text { duktion konnten aufgrund mehrerer ähnlicher } \\
\text { Reallabore forciert werden. }\end{array}$ \\
\hline $\begin{array}{l}\text { Transfer } \\
\text { an an- } \\
\text { deren } \\
\text {,Ort }\end{array}$ & $\begin{array}{l}\text { Die Erkenntnisse aus den Reallabo- } \\
\text { ren wurden in wissenschaftlichen } \\
\text { und praxisorientierten Artikeln zu- } \\
\text { sammengefasst. Eine Verstetigung } \\
\text { in den Branchen „Handwerk“ und } \\
\text { "Einzelhandel“ wird bis } 2021 \text { ange- } \\
\text { strebt. }\end{array}$ & $\begin{array}{l}\text { Die Ergebnisse inklusive } \\
\text { möglicher Verfahrenswei- } \\
\text { sen und Instrumente sind } \\
\text { in einer Broschüre festge- } \\
\text { halten, die sich explizit an } \\
\text { kommunale Akteure richtet. }\end{array}$ & $\begin{array}{l}\text { Maßnahmen zur Förderung urbaner Produktion } \\
\text { wurden in einem Handbuch beschrieben. Außer- } \\
\text { dem informierten sich andere Städte und Akteure } \\
\text { zum Vorgehen. Ein direkter Transfer wird im Fol- } \\
\text { geprojekt für die Stadt Gelsenkirchen und den } \\
\text { Stadtteil Herne-Wanne bis } 2021 \text { angestrebt. }\end{array}$ \\
\hline $\begin{array}{l}\text { Transfer } \\
\text { in an- } \\
\text { deren } \\
\text { Kontext }\end{array}$ & $\begin{array}{l}\text { Das Thema „Fachkräftesicherung“ } \\
\text { wird zukünftig in einem dritten Re- } \\
\text { allabor aufgenommen. Ein Transfer } \\
\text { des Ansatzes von partizipativer } \\
\text { Governance, in dem die Reallabore } \\
\text { ein zentrales Element bilden, steht } \\
\text { aus. }\end{array}$ & $\begin{array}{l}\text { Ein Transfer hat nicht statt- } \\
\text { gefunden. }\end{array}$ & $\begin{array}{l}\text { Ein Transfer der Maßnahmen urbaner Produktion } \\
\text { auf den ländlichen Raum ist denkbar. }\end{array}$ \\
\hline
\end{tabular}

ten Handlungen haben sich auf die Form des generierten Wissens ausgewirkt bzw. haben die Generierung von Transformationswissen erschwert. Es wurde verstärkt kontextbezogenes System- und Orientierungswissen gewonnen, während das Transformationswissen über die Auswirkung von nachhaltigen Praktiken auf die Kommunen und Branchen noch aussteht (vgl. Kapitel 4.1).

\subsection{Langfristigkeit und Übertragbarkeit}

Die Verstetigung der erarbeiteten Lösungen hängt von der verfügbaren Zeit, finanziellen Ressourcen und der Leidenschaft der Akteure im Reallabor ab.

Um der Frage nachzugehen, inwieweit die Reallabore in den drei Projekten zum Aufbau von langfristigen Experimentier- und Lösungswegen beigetragen haben, wurden die zwei Dimensionen „Verstetigung“ und „Übertragbarkeit“ separat evaluiert.

Verstetigung: Ob einzelne Reallaboraktionen oder das Reallabor als Instrument langfristig übernommen werden, um eine Verstetigung zu erreichen, bleibt laut Stand der Forschung offen. In den hier betrachteten Projekten zeichnen sich lediglich Verstetigungstendenzen des ersten Aspektes (Empowerment und stabile Kooperationen) ab. Tabelle 5 fasst den Zeitraum und die Endergebnisse in den verglichenen Reallaboren zusammen.

In den Fällen von Bottrop 2018+ und ProUrban wurden die in den Reallaboren entwickelten Interventionen und Maßnahmen von bestehenden Strukturen und Organisationen aufgegriffen und über die Laufzeit der Reallabo- re hinaus eigenständig fortgesetzt (z. B. Gründungswettbewerb $^{3}$ ). In KuDeQua und ProUrban sind neue Organisationsstrukturen (Einrichtung eines Quartiersmanagements, Verein) entstanden, die die begonnenen Interventionen eigenständig weiterführen. Zum Experimentieren gehört auch, dass Experimente scheitern und Akteure so in einem Prozess lernen. Experimentiergeist in Kommunen und bei Wirtschaftsakteuren zu wecken, hat sich jedoch als schwierig erwiesen, da deren reguläre Arbeitsmuster kein Scheitern erlauben. Dies birgt die Gefahr, dass Lernprozesse auf passive Maßnahmen (Veranstaltungsreihen statt Entwicklung von umsetzungsreifen Geschäftsmodellen) beschränkt bleiben. Einige Versuche, langfristige Strukturen $\mathrm{zu}$ etablieren, sind durch mangelndes Interesse, fehlende Bereitschaft wichtiger Akteure (z. B. Eigentümer), Zeitmangel, fehlender Beteiligung oder Finanzierung weiterer Akteure fehlgeschlagen. So konnte beispielsweise in ProUrban (Reallabor 2) erst während der Folgeförderung ein gemeinsamer Verein zur Fortsetzung gestarteter Aktivitäten gegründet werden.

Übertragbarkeit: Der Grad der Übertragbarkeit der Ergebnisse wurde im Vergleich der drei Fallstudien auf Basis der Skalierung und der Transferaktivitäten geschätzt (vgl. Tabelle 6).

In allen drei Projekten wurden sowohl wissenschaftliche als auch praxisnahe Publikationen erstellt, aktuelle Ergeb-

\footnotetext{
3 https://www.senkrechtstarter.de/wettbewerb/ueberblick/ (21.05.2021).
} 
nisse auf den Webseiten veröffentlicht und Vorträge gehalten, um das Wissen weiterzugeben. Dadurch wurde und wird der Transfer an andere Orte möglich. Der Transfer in andere Kontexte hat in Bottrop durch die Eröffnung eines neuen Themenfeldes stattgefunden. In den anderen Projekten ist das bislang nicht der Fall. Auch die Skalierung auf einen größeren Betrachtungsraum oder eine höhere Ebene erfolgte in den Projektzeiträumen nicht. Erst im Folgeprojekt ProUrban II wurde der Fokus auf die Gesamtstadt Bochum gelegt. Da das Thema urbane Produktion momentan in Wissenschaft und Praxis vor dem Hintergrund der postulierten Notwendigkeit nutzungsgemischter Städte stark diskutiert wird, konnten die Reallabore (inter)nationale Aufmerksamkeit erregen, die Thematik grundsätzlich skalieren und Diskussionen an anderen Orten und in weiteren Kontexten entfachen.

Die Erfahrungen zeigen, dass das erzeugte Wissen in allen Projekten transferiert wurde. Die Verstetigung und Übertragbarkeit hängen jedoch von der Verbreitung der Ansätze, dem Interesse anderer Kommunen und weiterer Akteure sowie von unterschiedlichen Ressourcen ab, die nicht immer im Voraus planbar sind.

\subsection{Kontinuierliche Reflexion}

Die Mitwirkung aller Projektpartner im Reflexionsprozess ist erforderlich. Reflexion betrifft sowohl den Reallabor-Prozess als auch die Nachhaltigkeit der Experimente.

Der Vergleich der drei Fallstudien zu diesem Charakteristikum eines Reallabors stand unter der Fragestellung: Wie und von wem wurde der Reflexionsprozess geleitet und wer wurde daran beteiligt? Tabelle 7 fasst die angewandten Reflexionsmethoden, deren Komplexität und Akzeptanz in den drei Fallstudien zusammen.

In den betrachteten Projekten war eine Reflexion von Beginn an vorgesehen, die Methoden wurden durch die wissenschaftlichen Akteure vorgegeben. Dabei war zwischen Nachhaltigkeits- und Prozessevaluation zu unterscheiden. Die angewandten Instrumente unterscheiden sich stark im Grad der Komplexität und in der Beteiligung der Akteure. In Bottrop 2018+ und KuDeQua wurden komplexe Instrumente (etwa Balance Scorecard und Monitoring), die eine aktive Beteiligung der Zielgruppen vorsahen, bereits zum Zeitpunkt der Antragstellung (Projektkonzipierung) festgelegt. Solche Instrumente bieten den Vorteil, neben dem Prozess des Reallabors die Nachhaltigkeit der Experimente beispielsweise durch ausgewählte Indikatoren analysieren zu können, die Reallabore dahingehend zu bewerten und bei Fehlentwicklungen anzupassen. In Bottrop 2018+ war geplant, dass die Akteure ein Monitoringinstrument anwenden, um auch alleine Aussagen treffen zu können. Dieses wurde jedoch aufgrund der Komplexität nicht akzeptiert. Als Folge wurde die Reflexion durch teilnehmende Beobachtung und Prozessreflexion ersetzt. In ProUrban dagegen wurden die Instrumente erst im Laufe des Projekts entwickelt: Während im ersten Reallabor niedrigschwellige Methoden zur Prozessreflexion angewandt wurden, kam im zweiten Reallabor ein strategieorientierterer Ansatz mit der Business Model Canvas zur Entwicklung und Evaluation des Reallabors zum Einsatz. Eine Beteiligung der Zielgruppe fand in beiden Reallaboren jedoch sehr niedrigschwellig mit Kurzfragebögen und in Gesprächen statt.

Tabelle 7 Reflexionsmethoden und deren Akzeptanz

\begin{tabular}{llll}
\hline & Bottrop 2018+ & KuDeQua & ProUrban \\
\hline Methoden/ & Monitoring der Nachhaltigkeit von & Balance Scorecard Prozess (BSC) & Tagesabschlussprotokolle \\
Tools & Realexperimenten durch Wesentlich- & Grad der Komplexität: hoch & Grad der Komplexität: niedrig \\
& keitsanalysen und vordefiniertem & Durchführung: ausschließlich Wis- & $\begin{array}{l}\text { Durchführung: ohne Beteiligung der } \\
\text { Zielgruppe, lediglich im Team }\end{array}$ \\
& Indikatorenset & senschaft & Canvas-Methode zur Entwicklung des \\
& Grad der Komplexität: hoch & Canvas-Methode zur Entwicklung & zeiten Reallabors \\
Durchführung: der Zielgruppe vorge- & von Konzepten und der Abschätzbar- & zweiten \\
stellt; abgelehnt & keit zur möglichen Umsetzung & Grad der Komplexität: hoch \\
Teilnehmende Beobachtung an aus- & Grad der Komplexität: hoch & Durchführung: ohne Beteiligung der \\
gewählten Reallabor-Treffen und & Durchführung: ohne Beteiligung der & Zielgruppe \\
Veranstaltungen sowie Interviews & Zielgruppe & Feedback-Bögen als Fragebogen für \\
mit beteiligten Akteuren & Kurzfragebogen zur Evaluation der & Anwesende \\
Grad der Komplexität: niedrig & Veranstaltungen & Grad der Komplexität: mittel \\
Durchführung: ausschließlich Wis- & Grad der Komplexität: niedrig & Durchführung: Beteiligung der Ziel- \\
senschaft & Durchführung: im Team entwickelt, & gruppe \\
& aufgrund situationsspezifischer Fak- & Reflexion im Dialog \\
& toren jedoch nicht eingesetzt & Grad der Komplexität: niedrig \\
& Reflexion im Dialog & Durchführung: mit und ohne Beteili- \\
& Grad der Komplexität: niedrig & gung der Zielgruppe
\end{tabular}


Die Erfahrungen zeigen, dass eine detaillierte Konzipierung und Festlegung der Methoden seitens der Wissenschaft nicht ausreichend ist, um diese mit der zu beteiligenden Gruppe (Zivilgesellschaft und Wirtschaftsakteure) durchzuführen. Solche Methoden (Balance Scorecard-Prozess und Monitoring) eignen sich zuvorderst als Instrument für die interne Reflexion im Team. In Abhängigkeit von der Komplexität des jeweiligen Instruments kann eine zeitaufwendige Einarbeitung der zu beteiligenden Akteure erforderlich sein, die diese überfordern und somit zur Ablehnung führen kann.

Ein hoher Grad an Akzeptanz hat sich auch bei den niedrigschwelligen Instrumenten zur Prozessevaluation als Voraussetzung erwiesen. Ist ein/e Projektpartner/-in nicht bereit, z. B. detaillierte Tagesabschlussprotokolle zu führen, geht Wissen verloren, das nicht in den weiteren Prozess eingebracht werden kann. Gleiches gilt für die Teilnahme an den Befragungen. Wird die Relevanz und der daraus resultierende Mehrwert der Evaluation und Reflexion von Projektbeteiligten unterschätzt bzw. nicht erkannt oder werden die vorgesehenen wissenschaftlichen Methoden nicht akzeptiert, wird die Reflexion für das Gesamtprojekt erschwert. Besteht hingegen ein generelles Verständnis für den damit verbundenen Mehrwert, können auch komplexere Instrumente zur Reflexion der Nachhaltigkeit eingebracht und genutzt werden. In jedem Fall wird die größte Last der Reflexion von der Wissenschaft getragen (vgl. Tabelle 3).

\section{Diskussion der Ergebnisse aus den Reallaboren Bottrop 2018+, KuDeQua und ProUrban}

In diesem Beitrag wurden die drei Forschungsprojekte Bottrop 2018+, KuDeQua und ProUrban verglichen, in denen Reallabore die zugrundeliegenden Instrumente waren. Als geeignete Vergleichsbasis haben sich die fünf Charakteristika von Schäpke, Stelzer, Bergmann et al. (2017) - Transformation, Transdisziplinarität, Experimente, Langfristigkeit und Übertragbarkeit, Kontinuierliche Reflexion - erwiesen. Jedoch hat der Vergleich gezeigt, dass eine vollständige Evaluation von Transdisziplinarität eine Unterscheidung zwischen Zusammenarbeit im Projektteam und im Reallabor als solchem verlangt. Diese Unterscheidung ist von der Logik der Forschungsprojekte abzuleiten, in denen das Reallabor-Vorhaben oft im langen Vorlauf zur Umsetzung (durch ein Projektteam) konzipiert wird. Weiterhin zeigt sich auch eine Unterteilung des Charakteristikums „Langfristigkeit“ in Evaluation der Verstetigung und der Übertragbarkeit als hilfreich. Zudem ist auch ein Dualismus in Bezug auf die Reflexion von Reallaboren zu beobachten. Um dem Anspruch auf Generierung von Transformationswissen gerecht zu werden, sollten der Reallabor-Prozess und die Nachhaltigkeit der Experimente getrennt und, wenn nötig, mit unterschiedlichen Instrumenten reflektiert werden. Solche Verfeinerungen der Charakteristika können zukünftige Vergleiche von Reallaboren erleichtern.

Inhaltlich hat der Vergleich gezeigt, dass:

- Reallabore die „Große Transformation“ nicht erreichen können, aber in Nischen zur Transition von Teilsystemen beitragen können,

- Reallabore Wissenschaft und Praxis vor neue Herausforderungen stellen, die nur durch hohe Flexibilität und Wahrnehmung unterschiedlicher Rollen aller Beteiligten überwunden werden können,

- der Unterschied zwischen Experiment und Maßnahmen oft unklar bleibt, was wiederum das Erzeugen von Transformationswissen erschwert,

- die Verstetigung der erarbeiteten Lösungen von Zeit (Defila/Di Giulio 2018; Parodi/Waitz/Bachinger et al. 2020), Geld (insbesondere für die aktive Umsetzung) und Leidenschaft der Akteure im Reallabor abhängt und transformative Lernprozesse Teil der Experimente sind (Parodi 2020) sowie

- die Mitwirkung aller Projektpartner/-innen im Reflexionsprozess erforderlich ist. Die Reflexion betrifft dabei sowohl den Reallabor-Prozess als auch die Nachhaltigkeit der Experimente.

In Bezug auf den Aspekt der Transformation hat sich gezeigt, dass Reallabore ein geeignetes Instrument sind, um System- und Orientierungswissen zu erzeugen und somit Transitionen anzustoßen. Dieses Wissen gilt es weiter zu operationalisieren. Transformationswissen konnte in den drei Projekten nur bedingt generiert werden. Zukünftig ist daher zu berücksichtigen, dass Projekte, die den Ansatz der Reallabore nutzen, eine längere Vorbereitungs- bzw. Konzeptphase vorsehen sollten, in der auch die zu beteiligenden Zielgruppen (Bürger/-innen oder Wirtschaftsakteure) aktiv eingebunden sind. Dies kann die Akzeptanz und die Bereitschaft, sich aktiv einzubringen, erhöhen und zugleich eine ,Überforschung` des Quartiers und der Bürger/-innen verhindern. In dieser Phase sollten sich alle Beteiligten (Team und Zielgruppe) kennenlernen und Vertrauen aufbauen. Eine so umfangreiche Vorbereitungsphase widerspricht unter Umständen der öffentlichen Förderlogik. Dies wirft die Frage auf, ob Reallabor-Projekte nicht eine andere, langfristig angelegte Förderung benötigen, damit die Erfolgschancen erhöht werden.

Darüber hinaus stellt der Reallabor-Prozess die Rolle der Wissenschaft in Frage. Die Fallstudien haben auch gezeigt, dass eine vordefinierte Rollenverteilung nicht zielführend erscheint, da der Reallabor-Prozess bestimmte personenbe- 
zogene Kompetenzen verlangt und zum Teil situativ und flexibel gehandelt werden muss. Diese Kompetenzen können sowohl bei wissenschaftlichen, aber auch bei kommunalen oder zivilgesellschaftlichen Akteuren verankert werden. Es gilt, diese Kompetenzen zu erkennen und das Team dahingehend zusammenzustellen, dass dieses über das entsprechende Portfolio an Fähigkeiten verfügt. Dabei kann es passieren, dass die wissenschaftlichen Partner/-innen die üblichen Rollen verlassen müssen, um die Praxispartner/-innen bei bestimmten Problemlagen zu unterstützen, wie beispielsweise bei dem Netzwerken oder der Aktivierung. Reallabore sind folglich durch ein kontinuierliches Aushandeln bezüglich Zielen, Aufgaben, Zuständigkeiten und Maßnahmen gekennzeichnet. Die Zusammenarbeit soll in allen Bereichen auf Augenhöhe erfolgen, um das Gefühl des ,beforschten Objekts' zu vermeiden und Ownership für nachhaltigkeitsspezifische Herausforderungen zu schaffen. Gleichzeitig ist es erforderlich, hinsichtlich Ausmaß der Beteiligung eine Balance zwischen zumutbar und wünschenswert zu finden. Während bei der Vorbereitung die Zielgruppe noch stärker zu beteiligen ist, sollte im Rahmen der Reflexion gut überdacht werden, welche Methoden für die Prozess- und Nachhaltigkeitsevaluation notwendig und umsetzbar sind. Es kann sich als zielführender erweisen, die Nachhaltigkeitsevaluation im Team oder von der Wissenschaft durchführen zu lassen.

Eine weitere Herausforderung für die Wissenschaft stellt die Art der Maßnahmen dar. Die Fallstudien machen deutlich, dass Maßnahmen oder einzelne Interventionen oft mit Experimenten gleichgesetzt werden. Reallabore müssten verstärkt Freiräume zur Erprobung von neuen Regulierungen, Gesetzen, Erneuerungen und ähnlichem bieten (Experimentierklauseln, Flexibilisierungsinstrumente; vgl. BMWi 2019). Der Vorteil von Reallaboren ist die hohe Toleranz und Anpassungsfähigkeit im Prozess. So können unterschiedliche Interventionen getestet, durch Reflexion überprüft und bei Bedarf angepasst werden. Dabei bedarf es in einigen Fällen einer hohen Frustrationstoleranz, um immer wieder von, vorn' zu beginnen, und zugleich bei gescheiterten Prozessen den Mut des Loslassens und Beschreitens neuer Wege. Wird dies im Projektteam unterstützt, können langfristig die auf die jeweilige Situation passenden Interventionen entwickelt und implementiert werden, wodurch eine Verstetigung aussichtsreicher wird. Reallaboren wird zunehmend eine Rolle als Katalysator für Prozesse zugeschrieben, die durch Verstetigung langfristig institutionalisiert werden können (Borner 2019: 356). Die Verstetigung wird Reallaboren zwar nicht immer als Funktion zugeschrieben, könnte aber in der Zukunft, im Sinne von politischen Rollen von Reallaboren in „Großen Trans- formationen“ von Bedeutung sein. ${ }^{4}$ Grundsätzlich liegen nun bereits viele Erfahrungen hinsichtlich der Reallabore sowie weitere Diskussionen bezüglich der dazugehörigen Charakteristika vor, weshalb sich weiterführende Forschung dahingehend anbietet.

\section{Welchen Pfad schlagen wir mit Reallaboren ein oder wohin geht die Reise?}

Einmal umgesetzt entwickeln Reallabore ein eigenes Leben und eine eigene Dynamik, die sich in der Regel von dem ursprünglichen (Forschungs-)Plan unterscheiden. Das bedeutet nicht zwangsläufig, dass die gesetzten Ziele nicht erreicht oder modifiziert werden müssen, sondern vielmehr, dass das Verhalten und Engagement der beteiligten Akteure nicht im Vorhinein absehbar ist. Die Umsetzung von Reallaboren ist von diesem Verhalten und Engagement stärker abhängig als von der theoretischen Planung zu Beginn. Letztendlich hängen Reallabore, neben der Investition von Zeit und Geld, in hohem Ausmaß von der Leidenschaft der beteiligten Akteure ab. Um diesem Faktor gerecht zu werden, sollten Reallabore als Methode so weiterentwickelt werden, dass sie Wissenschaft und Praxis gleichermaßen erlauben, das eigene Potenzial zu entfalten, Ideen mutig umzusetzen und diese auch zu verwerfen, wenn sie sich nicht als der richtige Weg darstellen. Hierzu gehört auch, Scheitern als Teil des Experimentes anzusehen und als Lernprozess zu verstehen, von dem alle Beteiligten profitieren können. Während dieser Vorgang der Forschung zugrunde liegt, ist er für die Praxispartner/-innen oft fremd und braucht Zeit, um Gehör $\mathrm{zu}$ finden und sich im täglichen Handeln zu verstetigen.

Förderhinweis Bundesministerium für Bildung und Forschung (BMBF), Förderprogramm „Nachhaltige Transformation Urbaner Räume“ (Bottrop 2018+ - FKZ: 01UR1612B; ProUrban - FKZ: 01UR1609A); Förderprogramm „Kommune innovativ“ (KuDeQua FKZ: 033L197B).

\section{Literatur}

Alcántara, S.; Lindner, D.; Löwe, C.; Kuhn, R.; Puttroweit, E. (2018): Die Kultur des Experimentierens. In Reallaboren Nachhaltigkeit gemeinsam schaffen. Stuttgart.

Arnstein, S. R. (1969): A Ladder of Citizen Participation. In: Journal of the American Institute of Planners 35, 4, 216-224. https://doi.org/10.1080/01944366908977225

Barth, M.; Michelsen, G. (2013): Learning for change: An

\footnotetext{
${ }^{4}$ Für diesen Hinweis bedanken wir uns bei einem der anonymen Gutachter.
} 
educational contribution to sustainability science. In: Sustainability Science 8, 1, 103-119. https://doi.org/10. 1007/s11625-012-0181-5

Beecroft, R.; Trenks, H.; Rhodius, R.; Benighaus, C.; Parodi, O. (2018): Reallabore als Rahmen transformativer und transdisziplinärer Forschung: Ziele und Designprinzipien. In: Defila, R.; Di Giulio, A. (Hrsg.): Transdisziplinär und transformativ forschen. Wiesbaden, 75-100. https://doi.org/10.1007/978-3-658-21530-9_4

BMWi - Bundesministerium für Wirtschaft und Energie (2019): Freiräume für Innovationen. Das Handbuch für Reallabore. Berlin.

Borner, J. (2019): Akzeptanz und Narrative - Kommunikationswissenschaftliche Perspektive. Reflexion der Erfahrungen in Reallaboransätzen in Mecklenburg-Vorpommern. In: Fraune, C.; Knodt, M.; Gölz, S.; Langer, K. (Hrsg.): Akzeptanz und politische Partizipation in der Energietransformation. Wiesbaden, 253-376.

Boschma, R. (2005): Proximity and innovation: a critical assessment. In: Regional Studies 39, 1, 61-74. https:// doi.org/10.1080/0034340052000320887

Caniglia, G.; Schäpke, N.; Lang, D. J.; Abson, D.J.; Luederitz, C.; Wiek, A.; Laubichler, M. D.; Gralla, F.; von Wehrden, H. (2017): Experiments and Evidence in Sustainability Science: A Typology. In: Journal of Cleaner Production 169, 5, 39-47. https://doi.org/10.1016/ j.jclepro.2017.05.164

De Flander, K.; Hahne, U.; Kegler, H.; Lang, D.; Lucas, R.; Schneidewind, U.; Simon, K.-H.; Singer-Brodowski, M.; Wanner, M.; Wiek, A. (2014): Resilience and Real-life Laboratories as Key Concepts for Urban Transition Research. In: GAIA - Ecological Perspectives for Science and Society 23, 3, 284-286. https://doi.org/10. 14512/gaia.23.3.19

Defila, R.; Di Giulio, A. (2018): Reallabore als Quelle für die Methodik transdiziplinären und transformativen Forschens - eine Einführung. In: Defila, R.; Di Giulio, A. (Hrsg.): Transdisziplinär und transformativ forschen. Wiesbaden, 9-35. https://doi.org/10.1007/978-3658-21530-9_1

Fung, A. (2006): Varieties of Participation in Complex Governance. In: Public Administration Review 66, s1, 66-75. https://doi.org/10.1111/j.1540-6210.2006.00667.x

Georg, A.; Quaas, W. (2018): Experimental turn. Das Reallabor im Kontext transformativer Forschung und Modus III-Wissenschaft. In: Raum und Resilienz 3, 1, 2-4.

Gonser, M.; Eckart, J.; Eller, C.; Köglberger, K.; Häußler, E.; Piontek, F. M. (2019): Unterschiedliche Handlungslogiken in transdisziplinären und transformativen Forschungsprojekten - Welche Risikokulturen entwickeln sich daraus und wie lassen sie sich konstruktiv einbinden? In: Defila, R.; Di Giulio, A. (Hrsg.): Transdiszi- plinär und transformativ forschen. Band 2. Wiesbaden, 39-83. https://doi.org/10.1007/978-3-658-27135-0_3

Grießhammer, R.; Brohmann, B. (2015): Wie Transformationen und gesellschaftliche Innovationen gelingen können. Dessau-Roßlau.

Grillitsch, M.; Trippl, M. (2014): Combining Knowledge from Different Sources, Channels and Geographical Scales. In: European Planning Studies 22, 11, 2305-2325. https://doi.org/10.1080/09654313.2013.835793

Groß, M.; Hoffmann-Riem, H.; Krohn, W. (2005): Realexperimente. Ökologische Gestaltungsprozesse in der Wissensgesellschaft. Bielefeld.

Grunwald, A. (2015): Transformative Wissenschaft - eine neue Ordnung im Wissenschaftsbetrieb? In: GAIA - Ecological Perspectives for Science and Society 24, 1, 17-20. https://doi.org/10.14512/gaia.24.1.5

Hens, L.; Nath, B. (2003): The Johannesburg Conference. In: Environment, Development and Sustainability 5, 1/2, 7-39. https://doi.org/10.1023/A:1025303511864

Howaldt, J.; Schwarz, M. (2010): Soziale Innovationen Konzepte, Forschungsfelder und -perspektiven. In: Howaldt, J.; Jacobsen, H. (Hrsg.): Soziale Innovation. Auf dem Weg zu einem postindustriellen Innovationsparadigma. Wiesbaden, 87-109. https://doi.org/10.1007/978-3531-92469-4

Jahn, D. (2011): Vergleichende Politikwissenschaft. Wiesbaden. https://doi.org/10.1007/978-3-531-92682-7

Kanders, M.; Rösner, E. (2006): Das Bild der Schule im Spiegel der Lehrermeinung. Ergebnisse der 3. IFS-Lehrerbefragung 2006. In: Jahrbuch der Schulentwicklung $14,11-48$.

Lang, D.J.; Wiek, A.; Bergmann, M.; Stauffacher, M.; Martens, P.; Moll, P.; Swilling, M.; Thomas, C.J. (2012): Transdisciplinary research in sustainability science: Practice, principles, and challenges. In: Sustainability Science 7, 1, 25-43. https://doi.org/10.1007/s11625011-0149-x

Lauth, H.-J.; Pickel, G.; Pickel, S. (2009): Methoden der vergleichenden Politikwissenschaft. Eine Einführung. Wiesbaden. https://doi.org/10.1007/978-3-658-08636-7

Lijphart, A. (1971): Comparative Politics and the Comparative Method. In: American Political Science Review 65, 3, 682-693. https://doi.org/10.2307/1955513

Lütje-Klose, B.; Urban, M. (2014): Professionelle Kooperation als wesentliche Bedingung inklusiver Schul- und Unterrichtsentwicklung. Teil 1: Grundlagen und Modelle inklusiver Kooperation. In: Vierteljahresschrift für Heilpädagogik und ihre Nachbargebiete 83, 2, 112-123. https://doi.org/10.2378/vhn2014.art09d

O'Hern, M.S.; Rindfleisch, A. (2010): Customer Co-Creation. In: Malhotra, N. K. (Hrsg.): Review of Marketing 
Research, Volume 6. Bingley, 84-106. https://doi.org/10. 1108/S1548-6435(2009)0000006008

Parodi, O. (2020): Erfahrungen aus der Reallabor-Arbeit mit Blick auf Verstetigung, Übertragung und Wirkungsverstärkung. Experimente in urbanen Reallaboren. Vortrag am 30. Juni 2020im Rahmen von SynVer*Z; Quer*Z. Karlsruher Institut für Technologie (KIT).

Parodi, O.; Beecroft, R.; Albiez, M.; Quint, A.; Seebacher, A.; Tamm, K.; Waitz, C. (2016): Von „Aktionsforschung“ bis „Zielkonflikte“. Schlüsselbegriffe der Reallaborforschung. In: Technikfolgenabschätzung - Theorie und Praxis 25, 3, 9-18. https://doi.org/10.14512/tatup. 25.3.9

Parodi, O.; Waitz, C.; Bachinger, M.; Kuhn, R.; MeyerSoylu, S.; Alcántara, S.; Rhodius, R. (2020): Erkenntnisse und Empfehlungen dreier Reallabore. Ein erfahrungsbasierter Vergleich. In: Quartier Zukunft (Hrsg.): Dein Quartier und Du. Nachhaltigkeitsexperimente im Reallabor zu Nachbarschaften, Bienen, Naschbeeten, Kreativität und Konsum. Karlsruhe, 275-289. https://doi.org/10. 5445/KSP/1000076132

Rose, M.; Wanner, M.; Hilger. A. (2019): Das Reallabor als Forschungsprozess und -infrastruktur für nachhaltige Entwicklung. Konzepte, Herausforderungen und Empfehlungen. Wuppertal. $=$ Wuppertal Paper 196.

Schäpke, N.; Stelzer, F.; Bergmann, M.; Singer-Brodowski, M.; Wanner, M.; Caniglia, G.; Lang, D. J. (2017): Reallabore im Kontext transformativer Forschung. Ansatzpunkte zur Konzeption und Einbettung in den internationalen Forschungsstand. Lüneburg. = IETSR Discussion Papers in Transdisciplinary Sustainability Research 1/2017.

Schäpke, N.; Stelzer, F.; Caniglia, G.; Bergmann, M.; Wanner, M.; Singer-Brodowski, M.; Loorbach, D.; Olsson, P.; Baedeker, C.; Lang, D. J. (2018): Jointly Experimenting for Transformation? Shaping Real-World Laboratories by Comparing Them. In: GAIA - Ecological Perspectives for Science and Society 27, S1, 85-96. https://doi.org/ 10.14512/gaia.27.S1.16

Schneidewind, U.; Augenstein, K.; Stelzer, F.; Wanner, M. (2018): Structure Matters: Real-World Laboratories as a New Type of Large-Scale Research Infrastructure: A Framework Inspired by Giddens' Structuration Theory. In: GAIA - Ecological Perspectives for Science and Society 27, S1, 12-17. https://doi.org/10.14512/gaia.27.S1. 5

Stauffacher, M.; Flüeler, T.; Krütli, P.; Scholz, R. W (2008): Analytic and Dynamic Approach to Collaboration: A Transdisciplinary Case Study on Sustainable Landscape Development in a Swiss Prealpine Region. In: Systemic Practice and Action Research 21, 6, 409-422, https://doi. org/10.1007/s11213-008-9107-7

Verba, S. (1967): Some Dilemmas in Comparative Research. In: World Politics 20, 1, 111-127. https://doi. org/10.2307/2009730

von Hauff, M.; Kleine, A. (2005): Methodischer Ansatz zur Systematisierung von Handlungsfeldern und Indikatoren einer Nachhaltigkeitsstrategie - Das Integrierende Nachhaltigkeits-Dreieck. Kaiserslautern. Technische Universität Kaiserslautern, Diskussionsbeitrag 19-05.

Walter, M. (2009): Participatory Action Research. In: Bryman, A. (Hrsg.): Social Research Methods. Oxford, 151-158.

Wanner, M.; Stelzer, F. (2019): Reallabore - Perspektiven für ein Forschungsformat im Aufwind. Wuppertal. = in brief 07/2019, Wuppertaler Impulse zur Nachhaltigkeit.

WBGU - Wissenschaftlicher Beirat der Bundesregierung Globale Umweltveränderungen (2011): Hauptgutachten. Welt im Wandel - Gesellschaftsvertrag für eine Große Transformation. Berlin.

WBGU - Wissenschaftlicher Beirat der Bundesregierung Globale Umweltveränderungen (2016): Der Umzug der Menschheit: Die transformative Kraft der Städte. Hauptgutachten. Berlin.

Welschhoff, J.; Terstriep, J.; Seipel, N.; Gonka, T. (2017): Resilienz, Nachhaltigkeit \& Transition als theoretische Leitplanken für das Projekt „Bottrop 2018+“. AP1.2 Bericht des Projekts „Bottrop 2018+ Auf dem Weg zu einer nachhaltigen und resilienten Wirtschaftsstruktur“. Gelsenkirchen. 